\section{Experience with Extended Infusion of Piperacillin-Tazobactam at a Teaching Hospital}

There has been increasing interest in the administration of ß-lactam antibiotics by either continuous or extended infusion as a means of achieving desired pharmacodynamic targets. In 2007, Lodise and others ${ }^{1}$ compared traditional dosing of piperacillin-tazobactam with an extended infusion regimen. Their results suggested improvements in terms of mortality and length of stay with the extended infusion for a subset of critically ill patients with infections due to Pseudomonas aeruginosa. In addition to improved patient outcomes, considerable cost savings were also realized at the authors' institution by using the extended infusion regimen. That article provided the impetus for our institution, a 1000-bed tertiary care teaching hospital, to undertake a pilot study to assess the feasibility of extended infusions. We encountered significant challenges during this pilot project, but even though our endeavour was ultimately unsuccessful, we identified a number of barriers that may be informative to other institutions attempting to incorporate extended infusions into their practice.

With the same daily dose, extended and continuous infusions of piperacillin-tazobactam prolong, to a similar extent, the period of time that the drug is above the minimum inhibitory concentration ( $\mathrm{T}>\mathrm{MIC}$ ) for the bacteria, which is desirable for the antimicrobial action of ß-lactam agents. ${ }^{2}$ The extended-infusion regimen used at our institution was selected to provide a 12-h infusion-free interval during which potentially incompatible IV medications could be administered. In addition, it was thought that the extended infusion might facilitate patient mobility relative to continuous infusions.

A proposal to administer piperacillin-tazobactam according to the regimen studied by Lodise and others ${ }^{1}$ was reviewed and supported by the hospital's Antimicrobial Subcommittee and was approved by the Pharmacy and Therapeutics Committee. The Medicine, Vascular, and Intensive Care units at 2 campuses of The Ottawa Hospital were selected for the pilot study, on the basis of the volume of piperacillin-tazobactam prescribed and the potential for patient benefit. The Pharmacy and Therapeutics Committee approved a pharmacist-initiated automatic substitution of orders for piperacillin-tazobactam $3.375 \mathrm{~g}$ IV $\mathrm{q} 6 \mathrm{~h}$ with piperacillintazobactam $3.375 \mathrm{~g} \mathrm{IV} \mathrm{q8h}$ infused over $4 \mathrm{~h}$ for the pilot wards. Support from nurse educators was obtained, and education of nurses, home care personnel, pharmacists, and medical staff followed. Information sheets were developed for patient charts and the nursing staff. Forms were provided to assess implementation of the new regimen for each patient and the need for additional venous access devices; clinical efficacy was not evaluated.

A 3-month pilot project was initiated in January 2008, but the original study period was extended because of poor compliance in completing the evaluation forms. In general, implementation was successful, with minimal feedback from nursing staff. According to the evaluation forms and periodic audits, patients were successfully converted to the extended infusion in $171(87 \%)$ of 197 cases reviewed. A small proportion were switched back to traditional dosing because of issues with IV access or compatibility, mobility, or patient refusal. The extended infusion was appropriately charted on the medication administration record in 117 (85\%) of 138 cases reviewed. In the cases where the 4-h infusion was not charted, nurses on the pilot wards indicated, when questioned, that they knew to infuse the drug over $4 \mathrm{~h}$.

Hospital-wide expansion of the program was planned for fall 2008 , but the expansion was subsequently postponed and the project ultimately discontinued because of ongoing issues and concerns. These problems included pharmacists' workload to convert or clarify orders; the presence of multiple computer entries for the various piperacillin-tazobactam dosing regimens, with resultant confusion for nurses; inconsistent charting of the notation "over 4 hours" in the medication administration record, which made it difficult to determine if the drug had been administered for the appropriate duration; missing or untranscribed order clarifications; and the potential for subtherapeutic dosing at other institutions in cases in which physicians ordered the $\mathrm{q} 8 \mathrm{~h}$ interval without the designation for extended infusion. However, the primary concern was an increase in the occurrence of medication incidents when physicians began ordering piperacillin-tazobactam "q8h" without the extended-infusion designation on nonpilot wards, including the emergency department, with delayed or missed clarification by pharmacists. Although some of these concerns would have been minimized by a hospital-wide program, the decision was made to revert to traditional dosing to avoid the potential for inadvertent subtherapeutic dosing.

Use of an extended infusion at a q6h interval could have reduced concerns about inadvertent administration with traditional (i.e., half-hour) infusion times. In a previous study, modelling of a regimen of $4.5 \mathrm{~g}$ q6h, with each dose infused over $3 \mathrm{~h}$, yielded acceptable target attainment (i.e., desired T $>$ MIC was reached for at least $90 \%$ of bacterial isolates tested). ${ }^{2}$ However, given the MIC distribution of Pseudomonas aeruginosa at The Ottawa Hospital, the regimen used in this pilot study, with q8h dosing and 4-h infusion, was predicted to yield $90 \%$ target attainment. As such, at this institution, administration of higher doses was not expected to offer additional clinical benefits and would have eliminated any advantage of the lower daily dosage and less frequent administration. Patients with neutropenia ${ }^{3}$ or critical illness ${ }^{4}$ may represent exceptions, although clinical data support use of the $3.375 \mathrm{~g} \mathrm{q} 8 \mathrm{~h}$ extendedinfusion regimen of piperacillin-tazobactam for critically ill patients with infections due to $P$. aeruginosa. ${ }^{1}$

In retrospect, a shorter pilot study with a broader population might have circumvented some of the problems related to continuity of care as it pertained to prescribing of piperacillin-tazobactam when patients were transferred between departments (e.g., from ICU to surgery). More aggressive education for prescribers might also have increased awareness of 
the purpose of this project and increased appropriate prescribing. Finally, errors related to prescribing, transcribing, and documenting administration may be minimized at institutions where physician order entry and automated medication administration records are used.

\section{References}

1. Lodise TP Jr, Lomaestro BM, Drusano GL. Piperacillin-tazobactam fo Pseudomonas aeruginosa infection: clinical implications of an extendedinfusion dosing strategy. Clin Infect Dis 2007;44(3):357-363.

2. Kim A, Sutherland CA, Kuti JL, Nicolau DP. Optimal dosing of piperacillin-tazobactam for the treatment of Pseudomonas aeruginosa infections: prolonged or continous infusions? Pharmacotherapy 2007;27(11):1490-1497.

3. Ariano RE, Nyhlén A, Donnelly JP, Sitar DS, Harting GKM, Zelenitsky SA. Pharmacokinetics and pharmacodynamics of meropenem in febrile neutropenic patients with bacteremia. Ann Pharmacother 2005;39(1): $32-38$
4. Roberts JA, Kirkpatrick CMJ, Roberts MS, Dalley AJ, Lipman J. First-dose and steady-state population pharmacokinetics and pharmacodynamics of piperacillin by continuous or intermittent dosing in critically ill patients with sepsis. Int J Antimicrob Agents 2010;35(2):156-163.

Rosemary Zvonar, BScPhm

Antimicrobial Pharmacy Specialist

The Ottawa Hospital

Ottawa, Ontario

Salmaan Kanji, PharmD

Critical Care Pharmacy Specialist

The Ottawa Hospital-General Campus

Associate Scientist

Ottawa Hospital Research Institute

Ottawa, Ontario

\section{Fancy yourself a photographer?}

The Canadian Journal of Hospital Pharmacy is bringing back a popular cover photo theme for 2010 :

\section{Canadian Landscapes and Scenery}

Interested participants are asked to submit a digital photo or group of photos for selection along with a short (max 150 words) write-up about the location of the photo, the date and time of the photo, and the type of equipment used. Be sure to include any stories or details that make this photograph or location unique!

Entries can be submitted to Colleen Drake, Publications Administrator, at cdrake@cshp.ca. 